\title{
THE EFFECTS OF A LOCAL INTERSTELLAR MAGNETIC FIELD ON VOYAGER 1 AND 2 OBSERVATIONS
}

\author{
Merav OpHeR \\ Department of Physics and Astronomy, George Mason University, 4400 University Drive, Fairfax, VA 22030; mopher@physics.gmu.edu \\ EDWARD C. STONE \\ California Institute of Technology, Pasadena, CA 91125; ecs@ srl.caltech.edu \\ AND \\ Paulett C. Liewer \\ Jet Propulsion Laboratory, California Institute of Technology, Pasadena, CA 91109; paulett.liewer@jpl.nasa.gov \\ Received 2005 December 30; accepted 2006 February 7; published 2006 March 6
}

\begin{abstract}
We show that an interstellar magnetic field can produce a north-south asymmetry in the solar wind termination shock. Using Voyager 1 and 2 measurements, we suggest that the angle $\alpha$ between the interstellar wind velocity and the magnetic field is $30^{\circ}<\alpha<60^{\circ}$. The distortion of the shock is such that termination shock particles could have streamed outward along the spiral interplanetary magnetic field connecting Voyager 1 to the shock when the spacecraft was within $\sim 2$ AU of the shock. The shock distortion is larger in the southern hemisphere, and Voyager 2 could be connected to the shock when it is within $\sim 5$ AU of the shock, but with particles from the shock streaming inward along the field. Tighter constraints on the interstellar magnetic field should be possible when Voyager 2 crosses the shock in the next several years.
\end{abstract}

Subject headings: interplanetary medium — ISM: kinematics and dynamics — solar wind — Sun: magnetic fields

\section{INTRODUCTION}

The motion of the solar system through the interstellar medium with a velocity of $25.5 \mathrm{~km} \mathrm{~s}^{-1}$ compresses the heliosphere, producing a comet-like shape with an extended tail. The heliosphere is created by the supersonic solar wind that abruptly slows, forming a termination shock as it approaches contact with the interstellar medium at the heliopause. Beyond the heliopause, the interstellar wind contains neutral atoms, mainly hydrogen and helium, and ions that carry the frozen-in interstellar magnetic field.

The twin Voyager spacecraft are probing the northern and southern hemispheres of the heliosphere. On 2004 December 16, Voyager 1 crossed the termination shock at $94 \mathrm{AU}$ and began exploring the heliosheath (Burlaga et al. 2005; Decker et al. 2005b; Stone et al. 2005). Voyager 1 is now beyond $98 \mathrm{AU}$ at 34.1 in latitude and $173^{\circ}$ in longitude, while Voyager 2 is beyond $78 \mathrm{AU}$ at $-26^{\circ} .2$ in latitude and $216^{\circ}$ in longitude (heliographic coordinates). In mid-2002, Voyager 1 began observing strong energetic beams of termination shock particles (TSPs) streaming outward along the spiral magnetic field upstream of the shock. Jokipii et al. (2004) and Stone et al. (2005) suggested that the upstream beaming resulted from a nonspherical shock with the spiral interplanetary magnetic field line crossing the shock (the source of TSPs) and returning to the supersonic solar wind before reaching Voyager 1.

An interstellar magnetic field oriented obliquely to the interstellar velocity can produce a lateral or north-south asymmetry in the heliospheric shape (Pogorolev \& Matsuda 1998; Ratkiewicz et al. 1998). However, the direction and intensity of the local interstellar magnetic field are not well constrained. Based on the polarization of light from nearby stars, Frisch (1990) suggested that the magnetic field direction is parallel to the Galactic plane (and directed toward $l \sim 70^{\circ}$ ). The source distribution of the Voyager $3 \mathrm{kHz}$ radio emission is also parallel to that plane (Kurth \& Gurnett 2003). On the other hand, Lallement et al. (2005) found from resonantly scattered solar Ly $\alpha$ radiation that the flow direction of interstellar neutral hydrogen differs from that of helium by $4^{\circ}$ in a direction consistent with an interstellar magnetic field plane inclined $60^{\circ}$ from the Galactic plane. This deflection has been demonstrated by model calculations (Izmodenov et al. 2005). In this Letter, we will assume that the interstellar field is parallel to this plane, which we will refer to as the H-deflection plane (HDP). There are few previous MHD models of the interaction of the solar and interstellar winds that include both the solar magnetic field and the interstellar magnetic field (Linde et al. 1998; Pogorolev et al. 2004; McNutt et al. 1999; Washimi \& Tanaka 2001).

In this Letter, we explore the nonspherical shape of the termination shock and the orientation of the interstellar magnetic field and show that observations from Voyager 1 and 2 may provide us with information on the inclination and strength of the interstellar magnetic field.

\section{RESULTS}

The model is based on the BATS-R-US code, a threedimensional magnetohydrodynamic (MHD) parallel, adaptive grid code developed by the University of Michigan (Gombosi et al. 1994) and adapted by Opher et al. $(2003,2004)$ for the outer heliosphere. We used a grid ranging from 1.5 to $20 \mathrm{AU}$ with an inner boundary at $30 \mathrm{AU}$ and outer boundaries at -1500 and $1500 \mathrm{AU}$ in the $y$ - and $z$-directions and at -800 and $800 \mathrm{AU}$ in the $x$-direction. The solar magnetic field axis was aligned with the solar rotation axis with a 26 day solar rotation period. The solar wind velocity was taken to be uniform at $450 \mathrm{~km} \mathrm{~s}^{-1}$; only the ionized component was included. The solar wind at the inner boundary was taken to have $n=$ $7.8 \times 10^{-3} \mathrm{~cm}^{-3}, T=1.6 \times 10^{3} \mathrm{~K}$, and a Parker spiral magnetic field of $2 \mu \mathrm{G}$ at the equator. For the interstellar wind, we used $n=0.07 \mathrm{~cm}^{-3}$ and $T=10^{4} \mathrm{~K}$ (Frisch 1996).

We considered interstellar magnetic fields in the HDP with different inclination angles $\alpha$, where $\alpha$ is the angle between the interstellar magnetic field and the interstellar wind velocity. 


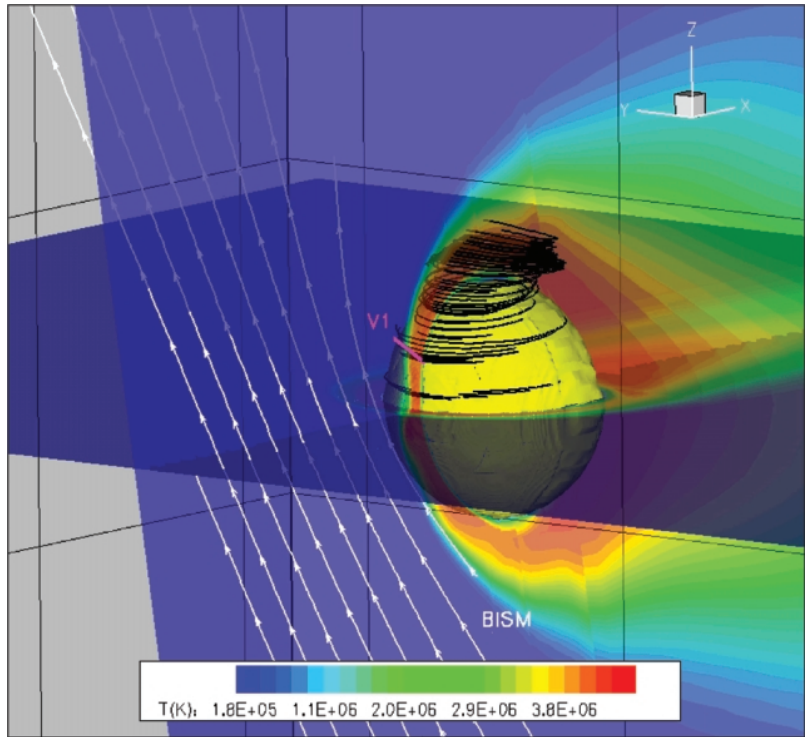

FIG. 1.-Model with $B_{\text {ISM }}$ in the HDP with $\alpha=90^{\circ}$ and $B_{\text {ISM, } y}<0$. The three-dimensional isosurface of the termination shock is denoted (yellow). The white lines are the interstellar magnetic field streamlines in the HDP, and the black lines are the solar magnetic field lines in the heliosheath. The trajectory of Voyager 1 is denoted (magenta). The contours denote temperatures in the plane containing Voyager 1 and the $x$-axis.

In the coordinate system, the interstellar velocity direction is in the $+x$-direction, and the $z$-axis is the solar rotation axis of the Sun, with $y$ completing the right-handed coordinate system. In this coordinate system, Voyager 1 is at $29^{\circ} .1$ in latitude and 178.4 in longitude, and Voyager 2 is at -31.2 in latitude and 213.4 in longitude, which ignores the 7.25 tilt of the solar equator with respect to the ecliptic plane.

Figure 1 shows the case for $B_{\text {ISM }}$ perpendicular to the flow $\left(\alpha=90^{\circ}\right) . B_{\text {ISM }}=1.8 \mu \mathrm{G}$ for Figures $1-5$. Subsonic flow carries the spiral solar field (black lines) into the hot heliosheath region (red) downwind of the termination shock (yellow surface). Only segments of the field lines appear in the nose region where the localized inward distortion of the shock results in field lines that cross into and back out of the heliosheath. Further from the nose, the solar magnetic field lines remain in the heliosheath as they spiral outward.

Figure 2 shows the contours of the magnetic field strength in the HDP for $B_{\mathrm{ISM}}$ with an inclination angle $\alpha=45^{\circ}$ and $B_{\text {ISM, } y}<0$. A large north-south asymmetry in the heliosheath is apparent as is the northward deflection of the heliospheric current sheet (HCS) in the heliosheath, which is thicker in the north (see also Pogorolev et al. 2004). The smaller north-south asymmetry in the termination shock will be discussed below.

The spiral solar magnetic field lines connecting to Voyager 1 and 2 lie on cones with latitudes of $29^{\circ} .1$ and -31.2 , and the intersections of those cones with the termination shock are shown in Figure 3. In both the northern and southern hemispheres, the cones intersect the surface of the termination shock closer to the equator, near the nose where the shock is closer to the Sun.

Figure 4 shows the intersection of the termination shock with the cones and the solar magnetic field lines for Voyager 1 and 2. The distortion of the shock is such that the shock is closer to the Sun counterclockwise from Voyager 1. It can be seen that $2 \mathrm{AU}$ inside the shock, Voyager 1 was connected to the shock along the field line in the direction toward the Sun, allowing TSPs to stream outward along the field as observed.

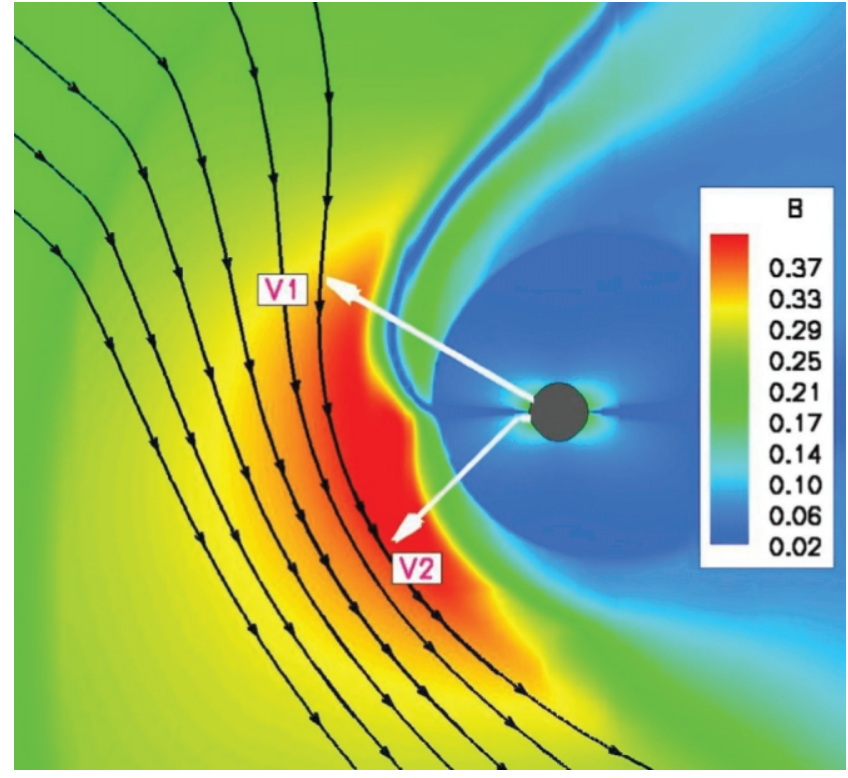

FIG. 2.-Contours of magnetic field strength $B(\mathrm{nT})$ in the HDP with $\alpha=45^{\circ}$ and $B_{\mathrm{ISM}, y}<0$. The black lines represent the interstellar magnetic field, and the white arrows denote the trajectories of Voyager 1 and Voyager 2. The heliospheric current sheet (deep blue) is deflected northward in the heliosheath.

The distortion is larger in the southern hemisphere, resulting in field lines 5 AU from the shock connecting the shock to Voyager 2. Also, the distortion is such that the shock is closer to the Sun clockwise from Voyager 2, so the TSP streaming should be inward along the spiral magnetic field line, opposite to that for Voyager 1, as has been observed (Cummings et al. 2005; Decker et al. 2005a).

The radial distance of the shock at Voyager 1 and 2 decreases significantly as the inclination angle $\alpha$ increases from $0^{\circ}$ to $60^{\circ}$, with the shock 7-10 AU closer in the south at Voyager 2 than in the north at Voyager 1 (Fig. 5, left). The maximum

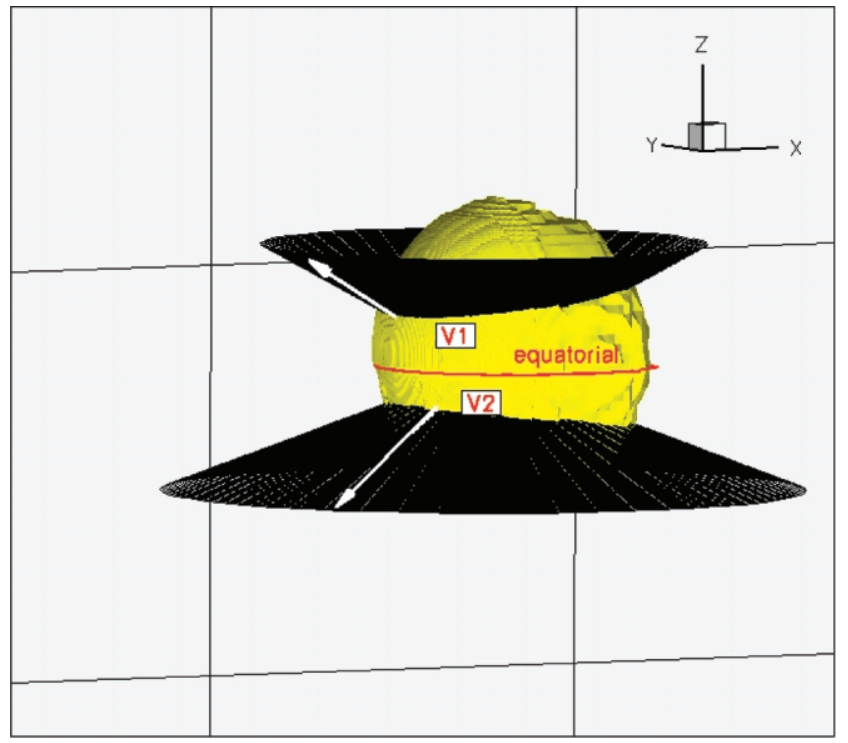

FIG. 3.-Intersection of the termination shock surface (yellow) with the cone of the interplanetary field line connecting to Voyager 1 and Voyager 2 (black lines). The trajectories of Voyager 1 and Voyager 2 are denoted as white arrows, and the solar equatorial plane is denoted as a red line. $B_{\mathrm{ISM}}$ is in the HDP with $\alpha=45^{\circ}$ and $B_{\text {ISM, } y}<0$. 

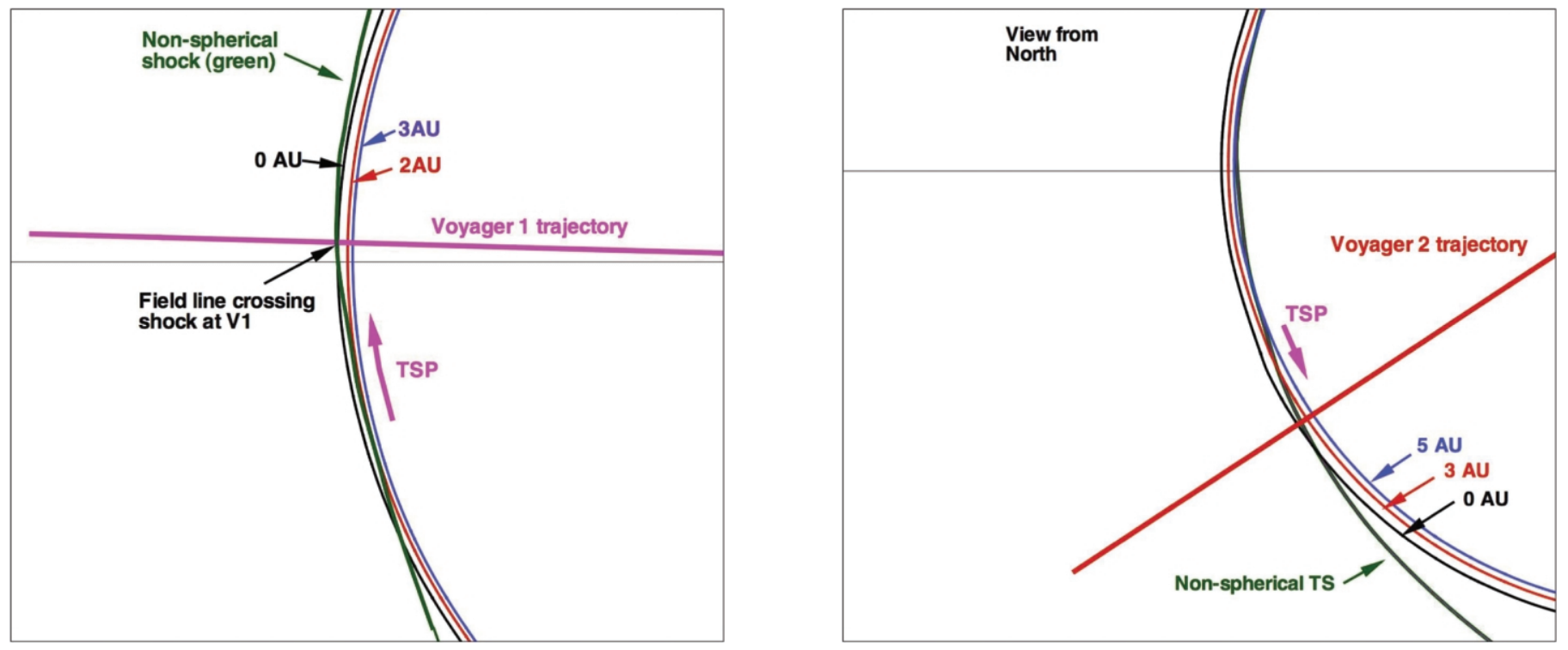

FIG. 4.-Expanded views from above of the field line cones. The plane of the plot is the surface of the interplanetary field line cones, the horizontal line is the intersection of the conical surface with the $x-z$ plane, and the green line indicates the intersection of the termination shock with that conical surface. Left: Spiral magnetic field lines on the Voyager 1 cone; the field line intersecting the shock where Voyager 1 crosses it is labeled 0 AU (black), with red and blue indicating, respectively, magnetic field lines 2.0 and 3.0 AU upwind from the $0 \mathrm{AU}$ line. The red arrow indicates the streaming direction of the termination shock particles from the shock along the field line to Voyager 1. Right: Similar to the left plot, but for Voyager 2, showing field lines 3.0 and 5.0 AU upwind of the 0 AU line. Note that in both views, the interplanetary magnetic field spirals outward with distance increasing clockwise.

distortion of the shock at Voyager 1 is $\sim 2.0$ AU (Fig. 5, right). Although there were no direct indications of how far Voyager $l$ was from the shock when upstream episodes of TSPs were observed, MHD models based on Voyager 2 solar wind pressure measurements (Richardson \& Wang 2005) suggest that the distance was less than 3-4 AU. This is somewhat larger than the maximum distortion in the right panel of Figure 5, suggesting that $\alpha$ is likely in the range of $30^{\circ}-60^{\circ}$, where the distortion is maximum.

The maximum distortion is much larger in the southern hemisphere and strongly depends on $\alpha$, with a maximum distortion at the latitude of Voyager 2 of 4-7 AU for $\alpha=45^{\circ}-60^{\circ}$. Thus, Voyager 2 should observe TSPs somewhat farther upstream from the shock than did Voyager 1. Because of the strong dependence on $\alpha$, the distance that TSPs are observed upstream of the shock by Voyager 2 should constrain the angle of inclination of the interstellar magnetic field.

The locations of the termination shock and heliopause scaled from various recent models are shown in Table 1, where the model results have been normalized to $90 \mathrm{AU}$, the estimated average location of the shock at the latitude of Voyager 1 as the shock moves inward and outward over the solar cycle. Some conclusions that can be drawn from Table 1 are as follows: (1) For the models that include an interplanetary magnetic field, the termination shock ranges between 76 and $82 \mathrm{AU}$ for Voyager 2. (2) There is somewhat less north-south asymmetry with a weaker $B_{\text {ISM }}$. (3) The heliosheath thickness on Voyager 1 is about 55-59 AU. For the model that has an interplanetary magnetic field and strong $B_{\mathrm{ISM}}$, the thickness at Voyager 2 is 33-45 AU. Although this is a preliminary calculation and does
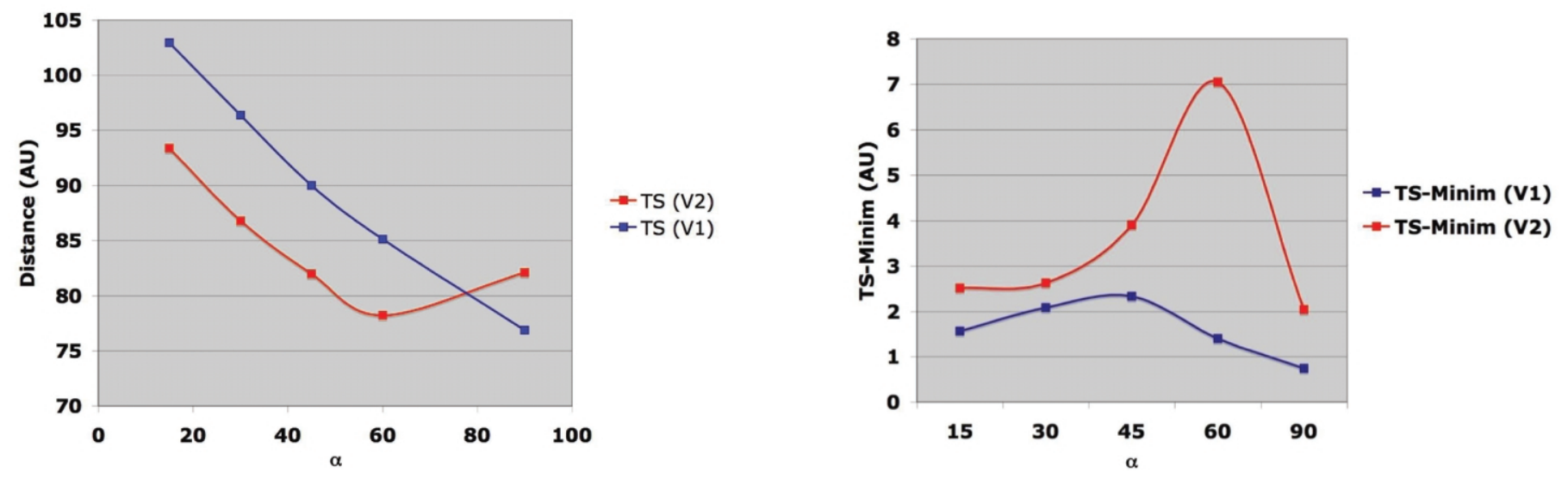

FIG. 5.-Left: Distance to the termination shock at Voyager 1 (blue line) and Voyager 2 (red line) latitudes as a function of the inclination angle $\alpha$ of the interstellar magnetic field. Right: Difference between the distance to the shock at the Voyager 1 and 2 longitude and the minimum distance to the shock as a function of $\alpha$ for Voyager 1 (blue line) and for Voyager 2 (red line). To match the estimated average shock location at Voyager 1, the shock locations have been normalized to $90 \mathrm{AU}$ for $\alpha=45^{\circ}$. In addition to these results for $B_{\mathrm{ISM}}=1.8 \mu \mathrm{G}$, a model was also run for $2.5 \mu \mathrm{G}$ and is summarized in Table 1 , along with locations determined from other recent model results. 
TABLE 1

COMPARISON OF MODELS

\begin{tabular}{|c|c|c|c|c|c|c|c|c|c|c|c|c|c|}
\hline Model & $\begin{array}{c}B \\
(\mu \mathrm{G})\end{array}$ & $P$ & $n$ & $B_{\mathrm{SW}}$ & $\alpha$ & $\begin{array}{c}\mathrm{TS} \\
(V 1)\end{array}$ & $\begin{array}{l}\text { HP } \\
(V 1)\end{array}$ & $\begin{array}{c}\mathrm{TS} \\
(V 2)\end{array}$ & $\begin{array}{l}\mathrm{HP} \\
(V 2)\end{array}$ & $\begin{array}{l}\text { HS } \\
(V 1)\end{array}$ & $\begin{array}{l}\text { HS } \\
(V 2)\end{array}$ & $\begin{array}{c}R \\
(\mathrm{TS})\end{array}$ & $\begin{array}{c}R \\
\text { (HP) }\end{array}$ \\
\hline Pogorolev et al. $2004 \ldots \ldots$. & 2.4 & G & Yes & Yes & 45 & 90 & 145 & 76 & 109 & 55 & 33 & 1.18 & 1.33 \\
\hline Izmodenov et al. $2005 \ldots \ldots$ & 2.4 & $\mathrm{H}$ & Yes & No & 45 & 90 & 146 & 91 & 139 & 56 & 48 & 0.99 & 1.05 \\
\hline \multirow[t]{2}{*}{ Our work $\ldots \ldots \ldots \ldots$. } & 2.5 & $\mathrm{H}$ & No & Yes & 45 & 90 & 149 & 79 & 124 & 59 & 45 & 1.13 & 1.20 \\
\hline & 1.8 & $\mathrm{H}$ & No & Yes & 45 & 90 & 145 & 82 & 132 & 55 & 50 & 1.10 & 1.10 \\
\hline Linde $1998 \ldots \ldots \ldots \ldots \ldots$ & 1.5 & $\mathrm{G}$ & Yes & Yes & 71 & 90 & 157 & 81 & 130 & 67 & 49 & 1.11 & 1.21 \\
\hline Our work (hydro) .......... & 1.8 & $\mathrm{H}$ & No & No & 45 & 90 & 130 & 87 & 127 & 40 & 40 & 1.03 & 1.02 \\
\hline
\end{tabular}

Notes. $-B$ is the magnitude of $B_{\text {ISM }}$ with inclination angle $\alpha ; P$ is the Galactic $(\mathrm{G})$ or the H-deflection $(\mathrm{H})$ plane of $B_{\text {ISM }} ; n$ indicates whether neutrals are included or not; $B_{\mathrm{Sw}}$ indicates whether the interplanetary magnetic field is included or not; TS is the distance to the termination shock at Voyager $1(V 1)$ and Voyager $2(V 2)$ normalized to the estimated average distance measured at Voyager 1; $\mathrm{HP}$ is the distance to the heliopause at $V 1$ and $V 2$; HS is the thickness of the heliosheath at $V 1$ and $V 2$; and $R$ is the ratio of the termination shock distances at $V 1$ and $V 2$ (TS) and the ratio of the heliosheath thicknesses (HS). In the last row, our model has no solar magnetic field.

not incorporate a tilted current sheet, it does indicate the possibility of a significant north-south asymmetry in the heliosheath thickness. (4) These models do not include neutrals that may affect the degree of asymmetry. However, models that included neutrals (see, e.g., Linde 1998) show a similar northsouth asymmetry with a ratio of $\sim 1.13$ for the north-south distance to the termination shock and 1.44 for the heliopause distances, similar to models without neutrals. Izmodenov et al. (2005), using a kinetic treatment with the $B_{\text {ISM }}$ in the HDP, found a smaller north-south asymmetry. Their treatment, however, did not include the solar magnetic field.

\section{CONCLUSIONS AND PREDICTIONS}

This model calculation indicates that an interstellar magnetic field $(B \sim 2 \mu \mathrm{G})$ in the $\mathrm{H}$-deflection plane can distort the he- liosphere in a manner consistent with the streaming observed by Voyager 1 and Voyager 2. The model also indicates that such a distortion will result in a significant north-south asymmetry in the distance to the shock and in the thickness of heliosheath. It is reasonable to expect that Voyager 2 will encounter the shock in the next several years, providing us with additional information about the strength and inclination of the local interstellar magnetic field.

The authors would like to thank the staff at NASA Ames Research Center for the use of the Columbia supercomputer. Part of this work is the result of research performed at the Jet Propulsion Laboratory of the California Institute of Technology under a contract with the National Aeronautics and Space Administration.

\section{REFERENCES}

Burlaga, L. F., Ness, N. F., Acuña, M. H., Lepping, R. P., Connerney, J. E. P., Stone, E. C., \& McDonald, F. B. 2005, Science, 309, 2027

Cummings, A. C., Stone, E. C., McDonald, F. B., Heikkila, B. C., Lal, N., \& Webber, W. R. 2005, AGU Fall Meeting, Abstract SH51A-1184

Decker, R. B., Krimigis, S. M., Roelof, E. C., \& Hill, M. E. 2005a, AGU Fall Meeting, Abstract SH43B-05

Decker, R. B., Krimigis, S. M., Roelof, E. C., Hill, M. E., Armstrong, T. P., Gloeckler, G., Hamilton, D. C., \& Lanzerotti, L. J. 2005b, Science, 309, 2020

Frisch, P. C. 1990, in Proc. First COSPAR Colloq., Physics of the Outer Heliosphere, ed. S. Grzedzielski \& D. E. Page (Oxford: Pergamon), 19 1996, Space Sci. Rev., 78, 213

Gombosi, T. I., Powell, K. G., \& de Zeeuw, D. L. 1994, J. Geophys. Res., 99, 21525

Izmodenov, V., Alexashov, D., \& Myasnikov, A. 2005, A\&A, 437, L35

Jokipii, J. R., Giacalone, J., \& Kota, J. 2004, ApJ, 611, L141

Kurth, W. S., \& Gurnett, D. A. 2003, J. Geophys. Res., 108, LIS 2-1

Lallement, R., Quémerais, E., Bertaux, J. L., Ferron, S., Koutroumpa, D., \& Pellinen, R. 2005, Science, 307, 1447
Linde, T. 1998, Ph.D. thesis, Univ. Michigan

Linde, T. J., Gombosi, T. I., Roe, P. L., Powell, K. G., \& DeZeeuw, D. L. 1998, J. Geophys. Res., 103, 1889

McNutt, R. L., Lyon, J., Goodrich, C. C., \& Wiltberger, M. 1999, in AIP Conf. Proc. 471, Solar Wind Nine, ed. S. R. Habbal et al. (Woodbury: AIP), 823

Opher, M., Liewer, P. C., Gombosi, T. I., Manchester, W., DeZeeuw, D. L., Sokolov, I., \& Toth, G. 2003, ApJ, 591, L61

Opher, M., et al. 2004, ApJ, 611, 575

Pogorelov, N. V., \& Matsuda, T. 1998, J. Geophys. Res., 103, 237

Pogorolev, N. V., Zank, G. P., \& Ogino, T. 2004, ApJ, 614, 1007

Ratkiewicz, R., Barnes, A., Molvik, G. A., Spreiter, J. R., Stahara, S. S., Vinokur, M., \& Venkateswaran, S. 1998, A\&A, 335, 363

Richardson, J. D., \& Wang, C. 2005, AGU Fall Meeting, Abstract SH51A1186

Stone, E. C., Cummings, A. C., McDonald, F. B., Heikkila, B. C., Lal, N., \& Webber, W. R. 2005, Science, 309, 2017

Washimi, H., \& Tanaka, T. 2001, Adv. Space Res., 27, 509 